SU-ITP-903

October 28, 1991

\title{
GEOMETRY OF SCATTERING AT PLANCKIAN ENERGIES
}

\author{
R. Kallosh円 \\ Physics Department, Stanford University \\ Stanford CA 94305
}

\begin{abstract}
We present an alternative derivation and geometrical formulation of Verlinde topological field theory, which may describe scattering at center of mass energies comparable or larger than the Planck energy. A consistent truncation of $3+1$ dimensional Einstein action is performed using the standard geometrical objects, like tetrads and spin connections. The resulting topological invariant is given in terms of differential forms.
\end{abstract}

\footnotetext{
${ }^{1}$ On leave of absence from: Lebedev Physical Institute, Moscow, 117924, USSR
} 
1. Recently a remarkable progress in understanding new aspects of $3+1$ dimensional gravity has been achieved by H. and E. Verlinde [1]. Their work sheds a new light on the results obtained by 't Hooft on scattering at center of mass energies comparable or larger than the Planck energy. In a series of his papers [2] it is shown that the high energy amplitudes have a universal behavior, similar to the behavior of two dimensional string amplitudes. This result has not been well understood and accepted by the scientific community.

Recent results of Verlinde stimulate new attempts to understand the whole complex of related ideas in a more deep way. They have found that the high energy scattering is described by a topological field theory, which is a gauge-fixed 3+1-dimensional Einstein theory, the quantization being performed under specific "high energy conditions". These conditions are attributed in [1] to the properties of the forward scattering at the center of mass energies comparable or larger than the Planck energy. In [1] the classical Lagrangian has been described in terms of metric. For performing the gauge fixing, however, it was necessary to introduce some vector fields in addition to metric. These vector fields are analogous to the fluid velocity in fluid mechanics. The final theory of high energy scattering presented in [1] is given in terms of a $3+1$ dimensional topological theory. The action, being a total derivative, is expressed through an integral over the boundary. The resulting 3-dimensional action is very simple, being quadratic in the dynamical variables. The physical field configuration is subject to the constraint that the vector fields are curl free in the $3+1$ dimensional space (in the absence of matter).

The theory of high energy scattering presented in [1] is extremely interesting. Unfortunately, however, the geometrical meaning of the additional vector fields introduced in [1] is somewhat obscure. In addition to this problem, the BRST quantization which has been performed in [1], was not really a standard one. After the gauge fixing, the action of ghost fields together with some part of the classical action (proportional to the constraint on vector fields) have been excluded from the final theory.

The purpose of the present paper is to represent the effective high energy theory in a completely geometrical way, without any problems with the gauge fixing. We will show that it is not necessary to perform BRST quantization in order to obtain the corresponding topological field theory under the high 
energy conditions used in [1]: It is quite sufficient to make a consistent truncation of the classical theory. We will start with 3+1-dimensional Einstein theory using tetrads and spin connections instead of metric. This will allow us to avoid introducing any auxiliary variables like the above mentioned vector fields. We are going to take another advantage by working in the so called 1.5 formalism [3, 4], where the spin connections $w$ are the functions of the tetrads $e$. The functions $w(e)$ are such that the equation of motion for spin connections is solved.

By the consistent truncation of a gauge theory we mean the following. We start with a gauge invariant action

$$
S(\phi, \Phi)
$$

depending on the set of gauge fields $\phi^{i}, \Phi^{a}$ with the gauge symmetry $\delta \phi^{i}=$ $R_{\alpha}^{i}(\phi, \Phi) \xi^{\alpha}$ and $\delta \Phi^{a}=R_{\alpha}^{a}(\phi, \Phi) \xi^{\alpha}$. One can perform the consistent truncation as follows. Suppose we want to exclude the fields $\Phi^{a}$. To make this consistent one must require that the classical field equation for these fields is satisfied. The new action is

$$
\tilde{S}(\phi) \equiv S(\phi, \Phi(\phi))
$$

where the function $\Phi^{a}(\phi)$ is a solution of the equation

$$
\frac{\partial S(\phi, \Phi)}{\partial \Phi^{a}}=0
$$

The action $\tilde{S}(\phi)$ defined by equation (2) depends on less variables than the original action (11). However, it is still gauge invariant under the gauge transformations of the smaller set of fields,

$$
\delta \tilde{S}(\phi)=\frac{\partial \tilde{S}(\phi)}{\partial \phi^{i}} R_{\alpha}^{i}(\phi, \Phi(\phi)) \xi^{\alpha}=0 .
$$

To prove that the truncated action is indeed symmetric, we can find its variation using eq. (2).

$\delta \tilde{S}(\phi)=\delta S(\phi, \Phi(\phi))=\left\{\frac{\partial S(\phi, \Phi)}{\partial \phi^{i}} R_{\alpha}^{i}(\phi, \Phi) \xi^{\alpha}+\frac{\partial S(\phi, \Phi)}{\partial \Phi^{a}} R_{\alpha}^{a}(\phi, \Phi) \xi^{\alpha}\right\}_{\mid \Phi=\Phi(\phi)}=0$. 
The expression in curly braces is equal to zero due to the invariance of the full action even before we substitute $\Phi(\phi)$ for $\Phi$. After this substitution the second term in curly braces vanish, and we are left with the first term only, which proves the gauge symmetry of the truncated action, expressed by eq. (四).

2. Consider the 3+1-dimensional Einstein-Hilbert action in the first order formalism,

$$
S^{1}=\int e^{m} \wedge e^{n} \wedge R^{p q}(w) \epsilon_{m n p q}
$$

where the tetrad forms $e^{m}=d x^{\mu} e_{\mu}^{m}$ are $S O(3.1)$ tangent space vectors, $m=$ $0,1,2,3, \quad \mu=0,1,2,3$. The curvature forms

$$
R^{p q}=(d w+w \wedge w)^{p q}
$$

are $S O(3.1)$ tangent space tensors. The spin connection forms $w^{p q}=d x^{\mu} w_{\mu}^{p q}$ are the connections for the local Lorentz group acting in $S O(3.1)$ tangent space. If the spin connections are independent variables in the Lagrangian in addition to tetrads, this is the first order formalism. The classical equation for spin connections is

$$
D e^{m} \wedge e^{n} \epsilon_{m n p q}=0,
$$

where the torsion-free condition

$$
T^{m}=D e^{m}=d e^{m}+w_{n}^{m} \wedge e^{n}=0
$$

solves equation (8). The solution to equations (8), (9) is given by

$w_{\mu m n}(e)=\frac{1}{2} e_{m}^{\nu}\left(\partial_{\mu} e_{n \nu}-\partial_{\nu} e_{n \mu}\right)-\frac{1}{2} e_{n}^{\nu}\left(\partial_{\mu} e_{m \nu}-\partial_{\nu} e_{m \mu}\right)-\frac{1}{2} e_{m}^{\rho} e_{n}^{\sigma}\left(\partial_{\rho} e_{p \sigma}-\partial_{\sigma} e_{p \rho}\right) e_{\mu}^{p}$.

One gets the second order formalism if one substitutes $w_{\mu m n}(e)$ from eq. (10) for $w_{\mu m n}$ in the Einstein-Hilbert action (6) and expresses the result as the function of tetrads only,

$$
S^{2}(e)=\int e^{m} \wedge e^{n} \wedge R^{p q}(w(e)) \epsilon_{m n p q} .
$$

This is the simplest example of the consistent truncation of the classical action described above. The action in the second order formalism (11) depends on the smaller set of fields than the action in the first order formalism 
(6). Still the action (11) has all gauge symmetries as the action (6): $3+1$ general covariance in the curved space and $S O(3.1)$ local Lorentz symmetry in the tangent space. All these symmetries are realized in terms of tetrads only. The proof that the consistently truncated theory (11) is generally and Lorentz covariant is a particular example of the proof given in eqs. (何), (5) for arbitrary theory.

The 1.5 formalism is a mixed case. It has been used for the first time by Fradkin and Vasiliev [3] in their discovery of $S O(2)$ supergravity. This formalism is described in detail in the review [4]. The 1.5 formalism action depends on tetrads and on spin connections. The spin connection, however, satisfies the equation of motion given in eq. (10).

$$
S^{1.5}(e, w(e))=\int e^{m} \wedge e^{n} \wedge R^{p q}(w(e)) \epsilon_{m n p q} .
$$

3. According to Verlinde, we choose our $x$-axis along the beam of high energy particles and introduce the notation $x^{\alpha} \equiv(t, x)$ for the longitudinal coordinates and $y^{i} \equiv(y, z)$ for the transversal coordinates. The momenta of particles in the $x^{\alpha}$-plane are of Planckian magnitude and the transversal momenta are negligible when we are dealing with forward scattering. The $3+1$-dimensional tangent space is also given by $S O(1.1)$ vectors $d x^{\mu} e_{\mu}^{a}=$ $e^{a}, \quad a=0,1$, and by $S O(2)$ vectors $d x^{\mu} e_{\mu}^{I}=e^{I}, \quad I=2,3$. The full exterior derivative operator $d$ now consists of longitudinal and transversal parts:

$$
d=d x^{\mu} \partial_{\mu}=d^{l g}+d^{t r}, \quad d^{l g}=d x^{\alpha} \partial_{\alpha}, \quad d^{t r}=d y^{i} \partial_{i} .
$$

Thus, all original indices are split as follows: $\mu=\alpha, i, \quad m=a, I$. With this geometric setup it is natural to try to perform a consistent truncation of the $3+1$ gravity to the smaller system without non-diagonal terms in tetrads, i.e. to exclude from the theory the variables $e_{\alpha}^{I}$ and $e_{i}^{a}$ by solving equations of motions for them,

$$
\begin{aligned}
& \epsilon^{\alpha \nu \rho \sigma} \epsilon_{I n p q} e_{\nu}^{n} R_{\rho \sigma}^{p q}(w(e))=\epsilon^{\alpha \beta i j} \epsilon_{I a b J} e_{\beta}^{a} R_{i j}^{b J}+2 \epsilon^{\alpha i \beta j} \epsilon_{I K a b} e_{i}^{K} R_{\beta j}^{a b}=0, \\
& \epsilon^{i \nu \rho \sigma} \epsilon_{\text {anpq }} e_{\nu}^{n} R_{\rho \sigma}^{p q}(w(e))=\epsilon^{i j \alpha \beta} \epsilon_{a I b J} e_{j}^{I} R_{\alpha \beta}^{b J}+2 \epsilon^{i \alpha j \beta} \epsilon_{a b I J} e_{\alpha}^{b} R_{j \beta}^{I J}=0 .
\end{aligned}
$$

Note, that to get these equations we have varied the action $S^{1.5}(e, w(e))$ (12) taking into account its explicit dependence on tetrads. The variation over 
the dependence on the tetrads through spin connections $w(e)$ drops from the equations of motion since the corresponding term is

$$
\frac{\partial S(e, w(e))}{\partial w_{\mu}^{m n}} \frac{\partial w_{\mu}^{m n}}{\partial e_{\sigma}^{p}}=0 .
$$

This term vanishes since in the 1.5 formalism $w$ satisfies equation

$$
\frac{\partial S(e, w(e))}{\partial w_{\mu}^{m n}}=0 .
$$

We will consider the solutions of classical equations (14) at $e_{\alpha}^{I}=0$ and $e_{i}^{a}=0$. The truncated action is given in terms of truncated tetrads (diagonal zweibeins ) and truncated spin connections. The truncated tetrads are:

$$
e_{\text {trun }}^{m}=\left\{e^{a}=d x^{\alpha} e_{\alpha}^{a}, \quad e^{I}=d y^{i} e_{i}^{I}\right\} .
$$

The truncated spin connections are functions of zweibeins $e_{\alpha}^{a}, e_{i}^{I}$, given by eq. (10) at $e_{\alpha}^{I}=0$ and $e_{i}^{a}=0$. The resulting truncated action is

$S_{\text {trun }}(e, w(e))=2 \int\left(e^{a} \wedge e^{b} \wedge R^{I J}(w(e))+e^{I} \wedge e^{J} \wedge R^{a b}(w(e))-2 e^{a} \wedge e^{I} \wedge R^{b J}(w(e))\right) \epsilon_{a b} \epsilon_{I J}$.

This truncation is consistent only when the truncated tetrads and connections satisfy classical eqs. (14). At this point we will add to the system described above the conditions which $\mathrm{H}$. and E. Verlinde attribute to the properties of the forward scattering at Planckian energies. Note, that until this point our treatment of the $3+1$ gravity which led us to eqs. (18), (14) was exact, no approximations have been done yet.

4. The high energy conditions derived in [1] in terms of metric variables are given by the equations

$$
\begin{aligned}
\partial_{\alpha} h_{i j} & =0, \\
R_{g} & =0,
\end{aligned}
$$

where $h_{i j}$ is the transverse metric $h_{i j}=e_{i}^{I} e_{j I}$ and $R_{g}$ is the scalar curvature of the longitudinal space build from the metric $g_{\alpha \beta}=e_{\alpha}^{a} e_{\beta a}$. The solutions 
to these conditions are taken in the form [1]

$$
\begin{aligned}
h_{i j} & =h_{i j}(y), \\
g_{\alpha \beta} & =\eta_{a b} \partial_{\alpha} X^{a} \partial_{\beta} X^{b} .
\end{aligned}
$$

We can reformulate both equations (19) in terms of our truncated tetrads (17) as one condition

$$
d^{l g} e_{\text {trun }}^{m}=0 \text {. }
$$

Note that the detailed form of this condition is

$$
d^{l g} e^{a}=d x^{\alpha} \partial_{\alpha} d x^{\beta} e_{\beta}^{a}=0, \quad d^{l g} e^{I}=d x^{\alpha} \partial_{\alpha} d y^{i} e_{i}^{I}=0 .
$$

The solution to high energy constraint (21) is

$$
\begin{aligned}
e^{a} & =d^{l g} X^{a}\left(x^{\alpha}, y^{i}\right) \\
e_{i}^{I} & =e_{i}^{I}\left(y^{j}\right)
\end{aligned}
$$

where $X^{a}$ is some $S O(1.1)$ tangent vector zero form.

5. Our next step is to constrain our consistent truncated action (18), with the variables satisfying eqs. (14), by the high energy constraint (21). This is quite straighforward. We will look for the spin connections which simultaneously solve the following system of equations:

i) the consistency condition for the truncation of tetrads to diagonal zweibeins, given in equations (14)

ii) the torsion free condition (9), (10) for tetrads $e_{\mu}^{m}$ truncated to diagonal zweibeins, satisfying eq. (22) .

The solution to this system of equations is

$$
\begin{aligned}
w^{a b} & \equiv d x^{\alpha} w_{\alpha}^{a b}+d y^{i} w_{i}^{a b}=0 \\
w^{I J} & \equiv d x^{\alpha} w_{\alpha}^{I J}+d y^{i} w_{i}^{I J}=d y^{i} w_{i}^{I J}, \\
w^{a I} & \equiv d x^{\alpha} w_{\alpha}^{a I}+d y^{i} w_{i}^{a I}=d x^{\alpha} w_{\alpha}^{a I}=e^{I i} \partial_{i} e^{a}=d^{l g} e^{I i} \partial_{i} X^{a},
\end{aligned}
$$

where

$$
d^{l g} w^{a I}=\left(d^{l g}\right)^{2} e^{I i} \partial_{i} X^{a}=0 .
$$


In particular, our solution includes the condition $w_{i}^{a b}=0$. This is the exact counterpart to Verlinde constraint $\partial^{[\alpha} V_{i}^{\beta]}=0$ translated from the fluid mechanics language to the geometric language of spin connections. The only non-zero components of the zweibein-compatible spin connections consistent with the high energy constraint are

$$
\begin{aligned}
w_{i I J} & =\frac{1}{2} e_{I}^{j}\left(\partial_{i} e_{J j}-\partial_{j} e_{J i}\right)-\frac{1}{2} e_{J}^{j}\left(\partial_{i} e_{I j}-\partial_{j} e_{I i}\right)-\frac{1}{2} e_{I}^{k} e_{J}^{l}\left(\partial_{i} k e_{K l}-\partial_{l} e_{K k}\right) e_{i}^{K} \\
w_{\alpha a I} & =e^{I i} \partial_{i} e_{\alpha}^{a}=e_{I}^{i} \partial_{i} \partial_{\alpha} X^{a} .
\end{aligned}
$$

These solutions for spin connections imply that some components of curvature tensors vanish,

$$
R_{i j}^{a I}=R_{\alpha i}^{a b}=R_{\alpha \beta}^{a I}=R_{i \alpha}^{I J}=0,
$$

which solves eqs. (14).

Note, that the conditions

$$
w_{\alpha}^{a b}=w_{\alpha}^{a I}=w_{\alpha}^{I J}=0
$$

are the consequences of the high energy conditions and torsion-free condition. The vanishing of $w_{i}^{a b}$,

$$
w_{i}^{a b}=0,
$$

comes from the solution of classical equations (14) when the high energy conditions are are already imposed.

The curvature tensors which enter the truncated action (18) are the following. In the first term we have the curvature of the transverse space

$$
R_{i j}^{I J}=R_{i j}^{I J}\left(w_{k}^{K L}\right) .
$$

In the second term of (18) we have

$$
R_{\alpha \beta}^{a b}=w_{\alpha I}^{a} w_{\beta}^{I b}-w_{\alpha I}^{b} w_{\beta}^{I a} .
$$

The third term contains

$$
R_{\alpha i}^{a I}=D_{i} w_{\alpha}^{b I}
$$


Now that the consistency condition for truncation has been solved with the high energy constraints being taken into account, the Einstein-Hilbert action has the same 3 terms as the truncated action:

$$
S_{\text {trun }}^{\text {Planck }}=2 \int\left(e^{a} \wedge e^{b} \wedge R^{I J}+e^{I} \wedge e^{J} \wedge R^{a b}-2 e^{a} \wedge e^{I} \wedge R^{b J}\right) \epsilon_{a b} \epsilon_{I J} .
$$

However, now we have in addition the consistency and the high energy conditions:

$$
d^{l g} e^{m}=d^{l g} w^{m n}=w^{a b}=d^{l g} R^{m n}=0, \quad m=0,1,2,3, \quad a=0,1 .
$$

The property of each term in this action to be a topological invariant follows from the simple fact that each term can be represented as $\int d^{l g}$ of something.

$S_{\text {trunc }}^{\text {Planc }}=2 \int d^{l g}\left\{\left(X^{a} \wedge e^{b} \wedge R^{I J}+e^{I} \wedge e^{J} \wedge e^{K i} \partial_{i} X^{a} w_{K}^{b}-2 X^{a} \wedge e^{I} \wedge R^{b J}\right) \epsilon_{a b} \epsilon_{I J}\right\}=T$.

In deriving equation (35) we have used eqs. (23), (24) and (31). Being a total divergence, the action (33) is still a gauge symmetric action: It is general covariant and Lorentz covariant for the gauge transformations which vanish at the boundary. This property is in a complete agreement with our definition of a consistent truncation of a gauge theory. The consistently truncated gauge action must be gauge symmetric, and it is gauge symmetric in our case!

6. If we denote the boundary values of $X^{a}$ by $\bar{X}^{a}$, the $3+1$ dimensional topological term (35) can be presented as an integral over the 3 -dimensional boundary,

$$
T=\int d \tau \int d V^{t r} \epsilon_{a b} \dot{\bar{X}}^{a}(\triangle-R)_{t r} \bar{X}^{b},
$$

where $\tau$-time parametrizes the coordinates $x^{\alpha}(\tau)$ on the boundary and $d V^{t r}$, $\triangle_{t r}$ and $R_{t r}$ are the volume of integration, the scalar Laplacian and curvature in the transversal $y$-space. This is the action derived in [1]. This action upon quantization leads to the fundamental equal- $\tau$-time commutation relations

$$
\left[X^{a}\left(y_{1}\right), X^{b}\left(y_{2}\right)\right]=i \epsilon_{a b} f\left(y_{1}, y_{2}\right),
$$

where $f$ is the Green function defined by the operator $(\triangle-R)_{t r}$. This commutator was suggested before by 't Hooft [2] and has been related to a new quantum gravitational uncertainty principle. 
Our present formulation allows to give a clear explanation of the origin of this commutator. We have started with the classical Einstein-Hilbert action and performed a completely consistent truncation of a gauge system. We have added the high energy constraint of Verlinde, which in our notations takes a very simple form (21), $d^{l g} e_{\text {trun }}^{m}=0$. The part of the classical truncated Einstein-Hilbert action which does not vanish when the constraint (21) is imposed, is given by the action (36), which leads to the commutation relations (37). Thus, eq. (21) is the only assumption behind the uncertainty principle discussed above.

In conclusion, we have derived the recent results of [1] in the geometric language, which is most appropriate for the description of the problem of the forward scattering at the Planckian energies.

It is a pleasure to thank J. Russo, L. Susskind and L. Thorlacius for valuable discussions. This work was supported in part by NSF grant PHY-

8612280 and by the John and Claire Radway Fellowship in the School of Humanities and Sciences at Stanford University.

\section{References}

[1] H. Verlinde and E. Verlinde, Princeton University preprint PUPT-1279 (1991).

[2] G. 't Hooft, Phys. Lett. B198 (1987) 61; Nucl. Phys. B304 (1988) 867; Nucl. Phys. B335 (1990) 138.

[3] E. S. Fradkin and M. A. Vasiliev, SO(2) Supergravity with Minimal Electromagnetic Interaction, 1.5-order Formalism and the Explicit Form of the Structure Coefficient, Lebedev Institute preprint No 197 (1976).

[4] P. van Nieuwenhuizen, Phys. Rep. 68 (1981) 189. 